\title{
Observing the Geometry of Warped Compactification via Cosmic Inflation
}

\author{
Gary Shiu and Bret Underwood \\ Department of Physics, University of Wisconsin, Madison, Wisconsin 53706, USA
}

(Received 20 October 2006; published 2 February 2007)

\begin{abstract}
Using Dirac-Born-Infeld inflation as an example, we demonstrate that the detailed geometry of warped compactification can leave an imprint on the cosmic microwave background. We compute cosmic microwave background observables for Dirac-Born-Infeld inflation in a generic class of warped throats and find that the results (such as the sign of the tilt of the scalar perturbations and its running) depend sensitively on the precise shape of the warp factor. In particular, we analyze the warped deformed conifold and find that the results can differ from those of other warped geometries, even when these geometries approximate well the exact metric of the warped deformed conifold.
\end{abstract}

DOI: 10.1103/PhysRevLett.98.051301

It has long been recognized that observational cosmology provides a powerful window for testing fundamental physics, especially theories whose energy scales are far from what current accelerator experiments can reach. In this regard, inflation is a particularly gratifying idea. In addition to the strong and growing experimental evidence in support of its basic picture, inflation significantly highlights the importance of microphysics. Quantum fluctuations in the early Universe are stretched by the enormous expansion of inflation to scales of astrophysical relevance, providing the seed for density perturbations. Thus, by way of inflation, short distance physics may leave an imprint on cosmological observables such as the cosmic microwave background (CMB) [1].

In recent years, significant progress has been made in constructing inflationary models from string theory. A particularly promising framework is the idea of brane inflation [2,3], in which the attraction between the branes gives rise to the inflaton potential. Advances in stabilizing moduli with fluxes $[4,5]$ have enabled the realization of this idea; some explicit models can be found in [6,7]. It is therefore worthwhile to explore if any stringy features, such as the geometry of compactification, can be determined or distinguished from precision cosmology.

In this Letter, we focus on the relativistic limit of brane inflation, known as Dirac-Born-Infeld (DBI) inflation [79], because of its strong observational potential. In particular, the primordial non-Gaussianity of the CMB in DBI inflation can be much larger than in slow roll models and is within the reach of current and next-generation CMB experiments like the Wilkinson Microwave Anistropy Probe (WMAP) and Planck [7,10]. More importantly, the shape of non-Gaussianities is distinct [10]. Thus, nonGaussianities of such size and shape, if observed, can put interesting bounds on the mass of the inflation which from [11] (see also [12]) may in turn constrain some gross features of the compact geometry.

A crucial element in the construction of brane inflation models is explicit solutions of warped throats, the most well studied example being the warped deformed conifold (or the Klebanov-Strassler (KS) throat) [13]. Besides sus-
PACS numbers: $98.80 . \mathrm{Cq}, 11.25 . \mathrm{Wx}$

taining inflation by flattening the inflaton potential [6] or limiting the speed of the branes [7], significant warping is essential in reheating the Universe [14]. Far from the tip of the throat and the bulk of the compact space (usually taken to be a Calabi-Yau manifold), the warped deformed conifold can be approximated by a product of $\mathrm{AdS}_{5} \times \mathrm{T}^{1,1}$ (up to a logarithmic correction [15]), where $T^{1,1}$ is a 5-dimensional Einstein-Sasaki manifold with the topology of $S^{2} \times S^{3}$. Therefore, as in [16], the warp factor can generate a hierarchy of scales. However, unlike an exact AdS throat, the warp factor approaches a constant near the tip which can be understood from AdS/CFT correspondence as a confining phase in the infrared of the dual gauge theory. In fact, the gauge-gravity duality suggests the existence of various other warped throats which are asymptotically AdS but differ in their geometries near the tip, though explicit metrics for such other warped geometries are not yet known at present. A question of significance is: could the precise geometry of warped throats be distinguished by observations?

The purpose of this Letter is to answer this question affirmatively. Naively, if the observed density perturbations leave the horizon in the AdS region of a warped throat (which, with the exception of [8], is assumed), small corrections to the geometry due, e.g., to the details of the tip region, are irrelevant. However, we will show, contrary to this expectation, that even if the last $55 e$-foldings of inflation takes place in the AdS region, small differences in the geometry can leave an observable effect.

Consider a 10 dimensional geometry with a warped throat whose metric takes the form,

$$
d s_{10}^{2}=f^{-1 / 2}(r) \eta_{\mu \nu} d x^{\mu} d x^{\nu}+f^{1 / 2}(r)\left(d r^{2}+d s_{X_{5}}^{2}\right)
$$

where $r$ is the proper distance from the tip of the throat, and $f(r)$ is the warp factor. The angular coordinates of $d s_{X_{5}}^{2}$ will not affect our analysis, and we will ignore them from now on.

The motion of a $D 3$-brane in a warped space is given by the DBI action, which in terms of the canonical scalar field $\phi=\mathrm{T}_{D 3}^{1 / 2} r$ and a rescaled warp factor $\tilde{f}(r)=\mathrm{T}_{D 3}^{-1} f(r)$ is 


$$
S_{\mathrm{DBI}}=-\int d^{4} x\left[\tilde{f}^{-1}\left(\sqrt{1-\tilde{f} \dot{\phi}^{2}}-1\right)-V(\phi)\right] .
$$

The factor inside the square root of Eq. (2) must always be positive, which leads to the speed limit $\dot{\phi}^{2}<\tilde{f}(\phi)^{-1}$ and a corresponding relativistic gamma factor $\gamma \equiv \frac{1}{\sqrt{1-\tilde{f} \dot{\phi}^{2}}}=$ $\sqrt{1+4 M_{p}^{4} H^{\prime 2} \tilde{f}(\phi)}$. DBI inflation occurs when the potential is steep, e.g., when the potential is dominated by a mass term $V(\phi)=m^{2} \phi^{2}$, which can arise for instance from moduli stabilization effects [11]. In this case, the Hubble parameter $H=m \phi\left(1-B \phi^{2}\right) / M_{p}$ where $B \phi^{2}$ represents small corrections coming from the kinetic term of the energy density [9], and we have chosen to absorb a factor of $\sqrt{3}$ into the definition of $m$. Notice that this implies $\gamma(\phi) \propto \tilde{f}^{1 / 2}(\phi)$ for relativistic $(\gamma \gg 1)$ motion.

The number of $e$-folds can be written as

$$
N_{e}=\int H d t=-\frac{1}{2 M_{p}^{2}} \int \frac{H}{H^{\prime}} \gamma(\phi) d \phi
$$

where $0000^{\prime}$ denote derivatives with respect to $\phi$. Irrespective of $\gamma$, this leads to the standard Lyth bound [17] $\frac{d \phi}{d N_{e}}=M_{p} \sqrt{\frac{r}{8}}$ where $r=16 \epsilon_{D} / \gamma$ is the tensor to scalar ratio.

The scalar spectral index and its running can be written as [9]

$$
\begin{aligned}
n_{s}-1 & =\frac{2 M_{p}^{2}}{\gamma}\left[-4\left(\frac{H^{\prime}}{H}\right)^{2}+2 \frac{H^{\prime \prime}}{H}+2 \frac{H^{\prime}}{H}\left|\frac{\gamma^{\prime}}{\gamma}\right|\right] \\
\frac{d n_{s}}{d \ln k} & =\frac{d}{d N_{e}} n_{s}=\frac{2 M_{p}^{2}}{\gamma} \frac{H^{\prime}}{H} \frac{d}{d \phi} n_{s}
\end{aligned}
$$

The first term of the spectral index is always negative and tends to make it red, while the last term is always positive and will tend to make it blue. The middle term is proportional to the small corrections of the kinetic term.

Given the metric of a warped throat, Eqs. (3) and (4) can in general be evaluated only numerically. We will present our numerical results for the KS throat, but to get an analytic idea that details of warped geometry can affect $\mathrm{CMB}$ observables, let us first consider a general warp factor of the form [8]:

$$
\tilde{f}(\phi)=\frac{1}{f_{0}+f_{2} \phi^{2}+f_{4} \phi^{4}}
$$

For such warp factor, the scalar spectral index Eq. (4) can be written (without any assumption for $\gamma=\sqrt{1+4 M_{p}^{4} H^{\prime 2} \tilde{f}}$ and $\left.H=m \phi / M_{p}\right)$,

$$
\begin{aligned}
n_{s}-1= & \frac{M_{p}}{m} \sqrt{\frac{f_{0}+f_{2} \phi^{2}+f_{4} \phi^{4}}{\left(1+\frac{f_{0}+f_{2} \phi^{2}+f_{4} \phi^{4}}{4 M_{p}^{2} m^{2}}\right)}}\left[-\frac{4}{\phi^{2}}\right. \\
& \left.+\frac{2\left(f_{2}+2 f_{4} \phi^{2}\right)}{f_{0}+f_{2} \phi^{2}+f_{4} \phi^{4}}\left(\frac{1}{1+\frac{f_{0}+f_{2} \phi^{2}+f_{4} \phi^{4}}{4 M_{p}^{2} m^{2}}}\right)\right]
\end{aligned}
$$

The differences between throats with different warped geometries can be seen readily from this equation. In particular, for a warped throat with a constant warp factor $\tilde{f}^{-1} \approx f_{0}$, the spectral index is red, while for an AdS-like throat $\tilde{f}^{-1} \approx f_{4} \phi^{4}$ the spectral index is slightly blue (the contributions in Eq. (6) cancel and the addition of extra corrections to the energy density coming from the kinetic terms leads to a slightly blue spectrum [9]).

Furthermore, the running of the scalar spectral index for $\gamma \gg 1$ [ignoring higher order terms in $H(\phi)$ ] can be written as

$$
\begin{aligned}
\frac{d n_{s}}{d \ln k}= & -\frac{2 M_{p}}{m} \\
& \times \frac{4 f_{0}^{2}+6 f_{0} f_{2} \phi^{2}+\left(f_{2}^{2}+8 f_{0} f_{4}\right) \phi^{4}+2 f_{2} f_{4} \phi^{6}}{\phi^{4}\left(f_{0}+f_{2} \phi^{2}+f_{4} \phi^{4}\right)} .
\end{aligned}
$$

The running is always negative except when $f_{0}=f_{2}=0$, in which case higher order corrections in $H$ need to be included. These corrections can lead to a small positive running in some cases. We see, then, that because the inflationary dynamics depend strongly on the warp factor through the speed-limiting behavior, details of the warped geometry can show up in the inflationary observables.

To illustrate this effect more clearly, let us now turn to explicit warped throats in string theory. For concreteness, we will consider two different warped throats and their corresponding warp factors: (i) AdS throat, with $\tilde{f}=\frac{\lambda}{\phi^{4}}$, cutoff at a fixed coordinate $\phi_{\text {cutoff }}=h_{\text {tip }} \lambda^{1 / 4} m_{s}$. (ii) $\mathrm{KS}$ throat, with $\tilde{f}=2^{2 / 3}\left(g_{s} M \alpha^{\prime}\right)^{2} \epsilon^{2 / 3} I[\tau(\phi)]$, where $M$ corresponds to the number of units of RR 3-form flux, $\tau(\phi)$ is a parameter along the radial direction of the throat, and $I(\tau(\phi))$ is an integral that can be determined numerically [13]. $\epsilon$ is related to the deformation of the conifold and is not to be confused with the inflationary parameter $\epsilon_{D}$. The KS geometry is sourced by a flux-induced $D 3$ charge, so the KS throat asymptotically matches the AdS solution far from the tip [13], see Fig. 1. For this reason, the AdS solution is often used as a simple model of the KS throat. Near the tip of the throat, the geometry is different, however, since the AdS throat is cut off at a finite value of the coordinate while the KS throat smoothly extends to $\phi=0$. It is precisely this difference that will become pronounced in the inflationary observables. The exact KS warp factor does not fit into our simple ansatz Eq. (5), and hence the corresponding inflationary observables have to be determined numerically. The "mass gap" warp factor $\tilde{f}=$ $\lambda\left(\mu^{2}+\phi^{2}\right)^{-2}$, where $\mu \propto \epsilon^{2 / 3}$ is related to the deformation of the tip, is of this form and can be shown to be a reasonably good approximation to the KS throat [8]. We can in principle compare the inflationary observables for a AdS throat with that of the mass gap warp factor to simplify our calculations. However, we wish to emphasize that the observable effects of the throat geometry are present in some explicit solutions of string theory, so we 


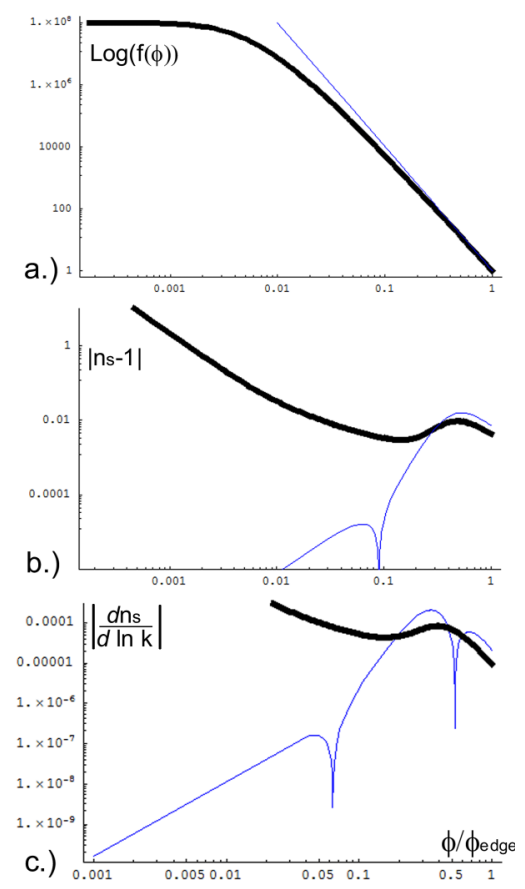

FIG. 1 (color online). (a) The warp factor for the AdS (thin line) and exact KS throat (thick line) are plotted as a function of the canonical scalar field scaled by its value at the edge where the throat is glued to the bulk space. We have chosen our parameters to be $h_{\text {tip }}=10^{-2}, M_{p}=100 m_{s}$ for these plots. (b) The absolute value of the spectral index for the two throats (thin line for AdS and thick line for KS). We take the absolute value of $n_{s}-1$ in order to show simultaneously the results for AdS and KS throat in one plot. The cusp in the AdS spectral curve corresponds to the tilt changing from negative at larger $\phi$ to positive for smaller $\phi$. This also happens where the warp factors begin to differ. (c) The absolute value of the running of the scalar spectral index is shown for the AdS (thin line) and KS (thick line) throats. The cusps in the AdS curve correspond to the running changing from negative to positive to negative again, as can easily be seen in the plot of the spectral index, see b.).

will carry out our numerical analysis for the exact KS metric.

For the KS throat, the first term in the spectral index in Eq. (4) dominates over the last, particularly near the tip where $\gamma^{\prime} \propto f^{\prime} \rightarrow 0$, so the spectral index is red. For the AdS throat, the last term is slightly bigger than the first term due to the fact that $\gamma^{\prime}$ keeps increasing near the tip, so the spectral index is blue near the tip, see Fig. 1. Note that the differences even become apparent when the deviation between the warp factors is small, so the details of the geometry can be important. The running of the scalar spectral index can also be computed (the tensor spectral index and its tilt are not significantly different), and one can see that the magnitude and sign of the running strongly depend on the geometry of the throat. We also show the spectral index for the two different throat geometries as a function of the number of $e$-folds in Fig. 2, where one sees that the region of the throat where the inflationary observables are different includes the last $60 e$-folds of inflation.

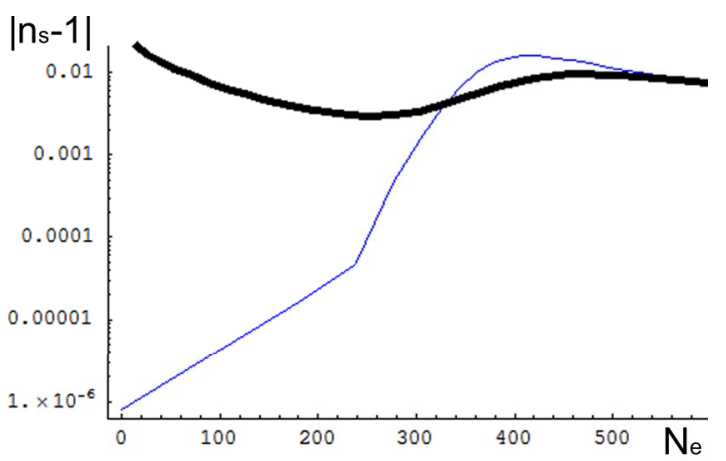

FIG. 2 (color online). The absolute value of the spectral index for the AdS (thin line) and KS (thick line) throats is shown as a function of the number of $e$-folds for $h_{\text {tip }}=10^{-2}$ and $M_{p}=$ $100 m_{s}$. The spectral index for AdS changes sign from negative to positive at the kinked point, so we see that the region where spectral index for the two throats is different falls within the last $60 e$-folds of inflation.

In order to make comparisons between these throats, one must compare the values of the spectral indices and their running when the observable modes cross the horizon. Inflation should end for the KS throat when stringy effects become important $\phi \sim \phi_{s}=h_{\text {tip }} m_{s}$ and for the AdS throat when the inflaton reaches the cutoff $\phi_{\text {cutoff }}=h_{\text {tip }} \lambda^{1 / 4} m_{s}$. We have (numerically) evaluated the inflationary observables $55 e$-folds back from these cutoffs for each throat, and the results are displayed in Fig. 3. It is then clear that the different throat geometries can have an observable effect on the spectral index and its running.

Incidentally, we have also found that the spectral index for DBI inflation in KS throat is red tilted (inclusion of the logarithmic correction term to the AdS throat as in [15] also leads to a red tilt). Although we have only shown our results for $n_{s}-1$ as a function of $h_{\text {tip }}$ in Fig. 3, the redness of the spectrum is robust against the change of the other parameter $m_{s} / M_{P}$. It is clear from Fig. 1 that the spectral index can be blue only for sufficiently small $\phi$. In this region of the throat, a scaling of $m_{s} / M_{P}$ is equivalent to a scaling of $h_{\text {tip }}$.

Finally, let us briefly comment on the observability of tensor modes. For the KS throat, we find that the tensor to scalar ratio $r$ is too small to be observed unless the inflaton moves Planckian distance as suggested by the Lyth bound. In contrast, the tensor signal is naively observable in an AdS throat for some ranges of parameters [9], although whether models with observable $r$ are under control within effective field theory is a subject of active investigation [18]. Nonetheless, it is clear that one should be cautious in using the AdS throat as an approximate description for more realistic warped geometries.

Our results thus provide a proof of concept that the shape of warped throats (as encoded by the warp factor) may be distinguishable by its effect on the inflationary observables. Interestingly, the same warped geometry can also leave an observable effect on particle physics observables, 

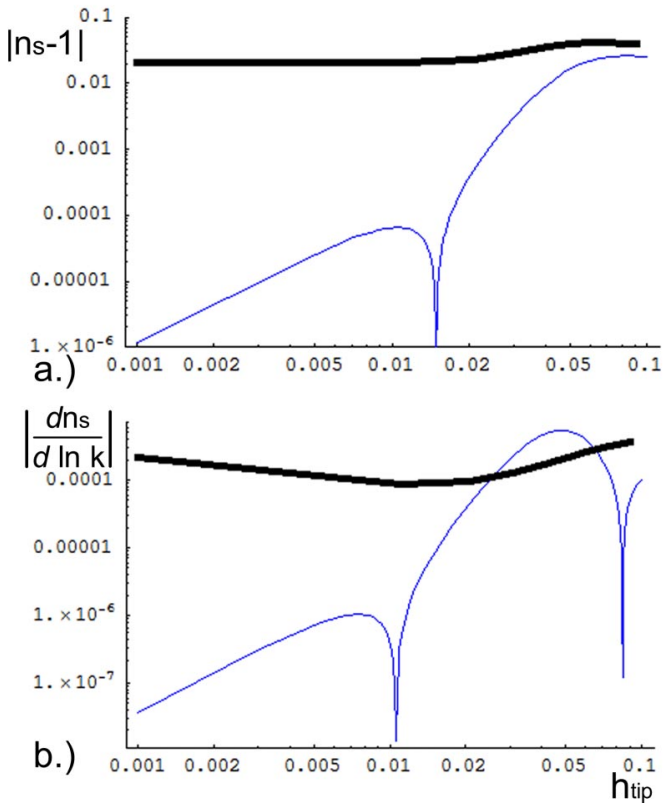

FIG. 3 (color online). (a) The absolute value of the scalar spectral index is shown for the AdS (thin line) and exact KS (thick line) throats as a function of the redshift factor at the tip $h_{\text {tip }}$ at $55 e$-folds back from the end of inflation defined in the text. We have fixed $M_{p}=100 m_{s}$. (b) The running of the spectral index also evaluated at $55 e$-folds back for the AdS and KS throats.

e.g., the pattern of soft supersymmetry breaking masses. Hence, combined data from particle physics and cosmology may allow us to decipher the underlying compactification geometry. While we explicitly demonstrated these differences by comparing the AdS and KS throats, it is straightforward to extend our analysis to other warped geometries as well. For example, it would be interesting to study the signatures of other throats, e.g., the baryonic branch of the KS solution [19] and other Einstein-Sasaki metrics such as the $Y_{p, q}$ [20], to learn how various warped throats may be distinguished from each other. Compactification effects may show up in the CMB for other stringy scenarios; some issues have been explored [21]. Work along these lines is underway.

We thank M. Huang, I. Klebanov, L. McAllister, and H. Tye for discussions. We have been informed of a forthcoming paper on related issues [22]. This work was supported in part by NSF CAREER Grant No. PHY-0348093, DOE Grant No. DE-FG-02-95ER40896, a Research Innovation grant and a Cottrell grant from Research Corporation.

[1] For a review, see, e.g., B. Greene, K. Schalm, J. P. van der Schaar, and G. Shiu, in 22nd Texas Symposium on Relativistic Astrophysics, edited by P. Chen et al., eConf C041213, 0001 (2004);D.P. Spergel et al., astro-ph/ 0603449, Section 5.3, and references therein.
[2] G. Dvali and S. H. H. Tye, Phys. Lett. B 450, 72 (1999).

[3] C.P. Burgess, M. Majumdar, D. Nolte, F. Quevedo, G. Rajesh, and R.J. Zhang, J. High Energy Phys. 07 (2001) 047; G. R. Dvali, Q. Shafi, and S. Solganik, hepth/0105203.

[4] S. Gukov, C. Vafa, and E. Witten, Nucl. Phys. B 584, 69 (2000); 608, 477(E) (2001); K. Dasgupta, G. Rajesh, and S. Sethi, J. High Energy Phys. 08 (1999) 023; T. R. Taylor and C. Vafa, Phys. Lett. B 474, 130 (2000); B. R. Greene, K. Schalm, and G. Shiu, Nucl. Phys. B 584, 480 (2000); G. Curio, A. Klemm, D. Lust, and S. Theisen, Nucl. Phys. B 609, 3 (2001); S. B. Giddings, S. Kachru, and J. Polchinski, Phys. Rev. D 66, 106006 (2002); K. Becker and M. Becker, J. High Energy Phys. 07 (2001) 038; S. Kachru, M. B. Schulz, and S. Trivedi, J. High Energy Phys. 10 (2003) 007.

[5] S. Kachru, R. Kallosh, A. Linde, and S. P. Trivedi, Phys. Rev. D 68, 046005 (2003).

[6] S. Kachru, R. Kallosh, A. Linde, J. Maldacena, L. McAllister, and S. Trivedi, J. Cosmol. Astropart. Phys. 10 (2003) 013.

[7] M. Alishahiha, E. Silverstein, and D. Tong, Phys. Rev. D 70, 123505 (2004); E. Silverstein and D. Tong, Phys. Rev. D 70, 103505 (2004); X. Chen, Phys. Rev. D 71, 063506 (2005); J. High Energy Phys. 08 (2005) 045.

[8] S. Kecskemeti, J. Maiden, G. Shiu, and B. Underwood, J. High Energy Phys. 09 (2006) 076.

[9] S. Shandera and S.-H. H. Tye, J. Cosmol. Astropart Phys. 05 (2006) 007.

[10] X. Chen, M. Huang, S. Kachru, and G. Shiu, hep-th/ 0605045.

[11] D. Baumann, A. Dymarsky, I. R. Klebanov, J. Maldacena, L. McAllister, and A. Murugan, J. High Energy Phys. 11 (2006) 031.

[12] M. Berg, M. Haack, and B. Kors, Phys. Rev. D 71, 026005 (2005).

[13] I. R. Klebanov and M. J. Strassler, J. High Energy Phys. 08 (2000) 052.

[14] G. Shiu, S. H. H. Tye, and I. Wasserman, Phys. Rev. D 67, 083517 (2003); N. Barnaby, C. P. Burgess, and J. M. Cline, J. Cosmol. Astropart Phys. 04 (2005) 007; L. Kofman and P. Yi, Phys. Rev. D 72, 106001 (2005); D. Chialva, G. Shiu, and B. Underwood, J. High Energy Phys. 01 (2006) 014; A. R. Frey, A. Mazumdar, and R. Myers, Phys. Rev. D 73, 026003 (2006); X. Chen and S. H. Tye, J. Cosmol. Astropart Phys. 06 (2006) 011.

[15] I. Klebanov and A. Tseytlin, Nucl. Phys. B 578, 123 (2000).

[16] L. Randall and R. Sundrum, Phys. Rev. Lett. 83, 3370 (1999).

[17] D. H. Lyth, Phys. Rev. Lett. 78, 1861 (1997).

[18] D. Baumann, J. Maldacena, and L. McAllister (to be published).

[19] A. Butti, M. Grana, R. Minasian, M. Petrini, and A. Zaffaroni, J. High Energy Phys. 03 (2005) 069; A. Dymarsky, I. R. Klebanov, and N. Seiberg, J. High Energy Phys. 01 (2006) 155.

[20] J.P. Gauntlett, D. Martelli, J. Sparks, and D. Waldram, Adv. Theor. Math. Phys. 8, 711 (2004); C. P. Herzog, Q. J. Ejaz, and I. R. Klebanov, J. High Energy Phys. 02 (2005) 009.

[21] J. Simon et al., astro-ph/0605371.

[22] R. Bean, S. Shandera, H. Tye, and J. Xu (to be published). 